\section{Medienpreis der Deutschen Allergologenverbände (MEDA 2008)}

Der Ärzteverband Deutscher Allergologen (ÄDA), die Deutsche Gesellschaft für Allergologie und klinische Immunologie (DGAKI) und die Gesellschaft für pädiatrische Allergologie und Umweltmedizin (GPA) vergeben zum vierten Mal den von ALK-SCHERAX im Rahmen der Initiative „Zukunft Allergologie“ gestifteten Medienpreis der deutschen Allergologenverbände (MEDA 2008). Der Medienpreis wird für hervorragende journalistische Arbeiten verliehen, die dazu beigetragen haben, der breiten Öffentlichkeit mehr Wissen über die Bedeutung, Vorbeugung und Diagnostik der Volkskrankheit Allergie zu vermitteln und die Versorgung von Allergikern in Deutschland zu verbessern. Die insgesamt mit 6.000,- Euro dotierten Preise werden im Rahmen des 3. Gemeinsamen Allergiekongresses verliehen, der vom 10. bis 13. September in Erfurt stattfindet.

\section{Zufrieden mit Hochgebirgsaufenthalt}

Die pneumologische und dermatologische Rehabilitation von Kindern und Jugendlichen an der Hochgebirgsklinik Davos hat einen lang anhaltenden Nutzen. Das zeigte eine im Rahmen der Qualitätssicherung durchgeführte Studie der höchstgelegenen deutschen Akutund Rehabilitationsklinik Europas. Auch nach bis zu sechs Jahren nach dem Klinikaufenthalt werden vom überwiegenden Teil der Befragten Besserungen in Bezug auf die Krankheit und ihre Folgen sowie ein hohes Ausmaß an Alltagstransfer angegeben. Die Ergebnisse bestätigen auch, dass durch individuelle und gezielte Förderung in Klinikschulen ein nahtloser Anschluss an die Heimatschulen gewährleistet werden kann. Auch die Zufriedenheit mit unterschiedlichen Aspekten des Klinikaufenthaltes war hoch. Die betroffenen Eltern gaben an, auch nach Jahren noch sehr zufrieden zu sein und würden bei Bedarf eine derartige Maßnahme in der Klinik wieder in Anspruch nehmen.

Die Ergebnisse bestätigen die Relevanz stationärer Rehabilitationsmaßnahmen in der Langzeitbehandlung chronisch kranker Kinder und Jugendlicher mit Erkrankungen der Atemwege, der Haut und Allergien.

Nach Presseinformationen der Hochgebirgsklinik Davos

\title{
Zuverlässiger Motor für Leitlinien
}

A m Rande des Mainzer AllergieWorkshops traf sich wie gewohnt die Arbeitsgruppe Nahrungsmittelallergie. Mitglieder der deutschen Allergiegesellschaften diskutierten gemeinsam mit österreichischen und schweize- über Besonderheiten bei atopischem Ekzem und chronischer Urtikaria sowie Konsequenzen durch Kreuzreaktionen und die Deklarationspflicht von potentiellen Nahrungsmittelallergenen bis hin zu therapeutischen Strategien. Frau

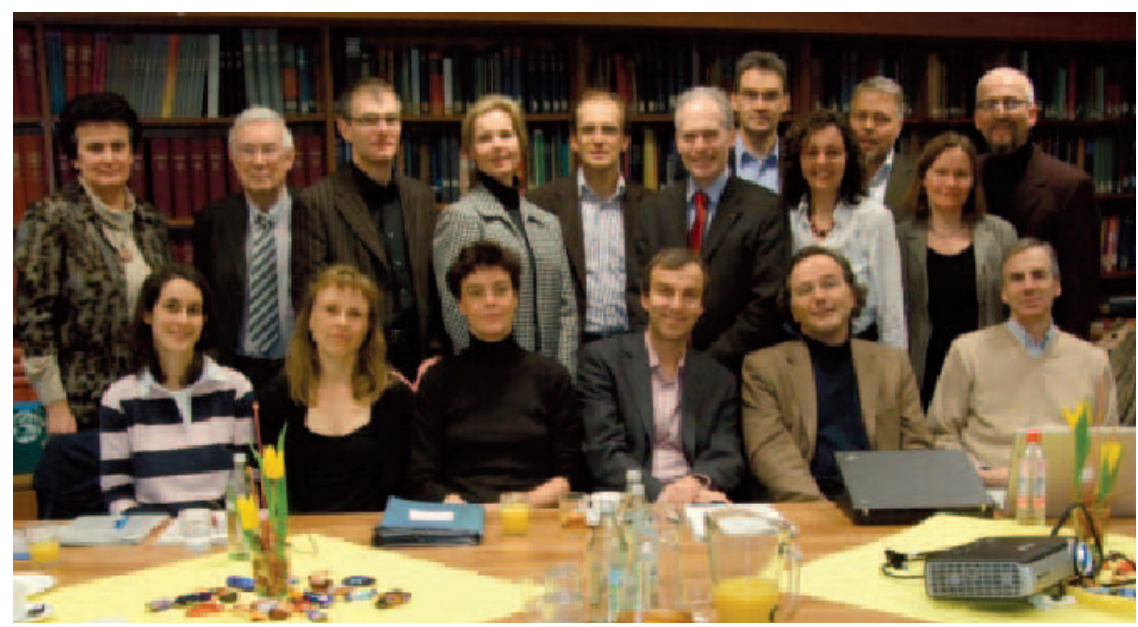

Die Teilnehmer des Treffens der AG Nahrungsmittelallergie im März 2008 von links nach rechts. Hinten: Priv.-Doz. Dr. Margot Henzgen, Prof. Dr. Lothar Jäger, Prof. Dr. Stefan Vieths, Priv.-Doz. Dr. Uta Jappe, Prof. Dr. Zsolt Szépfalusi, Prof. Dr. Torsten Zuberbier, Prof. Dr. Martin Raithel, Dr. Ute Lepp, Prof. Dr. Joachim Saloga, Dr. Kirsten Beyer, Priv.-Doz. Dr. Jörg Kleine-Tebbe; vorne: Dr. Annice Heratizadeh, Dr. Imke Reese, Prof. Dr. Margitta Worm, Prof. Dr. Thomas Werfel, Prof. Dr. Thomas Fuchs, Prof. Dr. Bodo Niggemann

rischen Kollegen neue und aktualisierte Leitlinien zum Thema Nahrungsmittelallergie. Seit dem Start mit Prof. Lothar Jäger und während der acht Jahre erfolgreicher Fortsetzung durch Prof. Thomas Werfel wurden alle wichtigen Themen der Nahrungsmittelallergie behandelt: Von der Diagnostik im Labor oder mit Hilfe der oralen Provokation
Prof. Margitta Worm koordiniert zukünftig die Arbeit der AG. So können Fortschritte auf dem komplexen Gebiet der Nahrungsmittelallergie gebündelt und zeitnah verbreitet werden.

Die aktuellen Leitlinien sind online verfügbar unter www.dgaki.de/Leit linien/leitlinien.html.

Priv.-Doz. Dr. Jörg Kleine-Tebbe, Berlin

\section{Ambrosia-Projekt begonnen}

A m 1. Mai 2008 hat das Projekt Ambrosia Cops JC FriedrichshainKreuzberg begonnen. Es verfolgt das Ziel, die Häufigkeit und Verbreitung von Ambrosia in Berlin zu reduzieren oder die Pflanze sogar ganz zu beseitigen. Das Allergie-Centrum-Charité und die Stiftung Deutscher Polleninformationsdienst (PID) unterstützten das Projekt Ambrosia Cops fachlich. Prof. Dr.
Torsten Zuberbier, Sprecher des Allergie-Centrum-Charité, Campus Mitte, und Prof. Dr. K.-Ch. Bergmann, Leiter der Stiftung Deutscher Polleninformationsdienst, rufen alle städtischen Organe dazu auf, das Projekt Ambrosia auf alle Stadtbezirke Berlins auszudehnen. FK

Nach Presseinformationen des AllergieCentrums-Charité 